\title{
Exercise-Induced Intranodal Atrioventricular Block
}

\author{
Mi-Youn Park, MD', Woo-Seung Shin, MD², Byung-Ju Shim, MD', Seung-Jae Lee, MD', \\ Jae-Hong Park, MD', Yoon-Seok Koh, MD², and Jong-Min Lee, MD² \\ ${ }^{1}$ Division of Cardiology, Department of Internal Medicine, Pohang St. Mary's Hospital, Pohang, \\ ${ }^{2}$ Division of Cardiology, Department of Internal Medicine, The Catholic University of Korea School of Medicine, Uijeongbu St. Mary's Hospital, Uijeongbu, Korea
}

Exercise-induced atrioventricular (AV) block in patients with normal AV conduction at rest is rare. Herein, we describe the case of a 67-yearold woman with normal $1: 1 \mathrm{AV}$ conduction at rest, who developed complete AV block during a treadmill test. Our patient complained of effort-related dizziness and dyspnea, which had been ongoing for 3 months. The patient's physical examination was normal. The resting electrocardiogram showed left anterior fascicular block with a PR interval of 0.19 seconds. The echocardiogram was normal except for mild aortic valve regurgitation. During the treadmill test, the patient developed complete AV block at a sinus rate of 90 beats $/$ min, which was followed by $2: 1$ AV block associated with dyspnea and dizziness. The patient's coronary angiogram was normal, and the ergonovine provocation test was negative. Electrophysiological studies demonstrated rate-dependent intranodal AV block. The patient received implantation of a permanent dual chamber (DDD) pacemaker and had no further symptoms during the follow-up period. (Korean Circ J 2012;42:698-701)

KEY WORDS: Atrioventricular block; Exercise test; Electrophysiologic techniques, cardiac.

\section{Introduction}

Normal electrocardiograms (ECGs) at rest rarely reveal exerciseinduced atrioventricular (AV) block, which has clinical implications in that such AV block may induce dyspnea and syncope. While most cases of exercise-induced AV block are induced by infranodal block, some cases with intranodal block can be induced by myocardial ischemia due to atherosclerotic changes or spasms of the coronary artery. There have been no reports of exercise-induced intranodal AV block without myocardial ischemia in Korea. Herein, we report a case of exercise-induced intranodal AV block. A brief review of the literature has been included.

Received: December 10, 2011

Revision Received: February 27, 2012

Accepted: March 5, 2012

Correspondence: Woo-Seung Shin, MD, Division of Cardiology, Department of Internal Medicine, The Catholic University of Korea School of Medicine, Uijeongbu St. Mary's Hospital, 271 Cheonbo-ro, Uijeongbu 480-717, Korea Tel: 82-31-820-3593, Fax: 82-31-841-7897

E-mail: medsws@catholic.ac.kr

- The authors have no financial conflicts of interest.

This is an Open Access article distributed under the terms of the Creative Commons Attribution Non-Commercial License (http://creativecommons. org/licenses/by-nc/3.0) which permits unrestricted non-commercial use, distribution, and reproduction in any medium, provided the original work is properly cited.

\section{Case}

A 67-year-old woman complained of a 3-month history of effortrelated dizziness and dyspnea. She had no significant medical history. Her physical examination revealed a regular pulse of 60 beats/ min and a blood pressure of 130/80 $\mathrm{mm} \mathrm{Hg}$. Chest radiography and routine laboratory tests were normal. The resting standard 12-lead ECG revealed PR interval of 190 msec and 1:1 AV conduction with left anterior fascicular block at a rate of 56 beats/min. The echocardiogram was normal except for mild aortic valve regurgitation. Treadmill exercise testing (modified Bruce protocol) was performed. During stage 1 of the test, her ventricular rate suddenly dropped to 50 beats/min, at an atrial rate of 90 beats/min, and her ECG showed variable degrees of AV block. During stage 2, she complained of dyspnea and dizziness with a maximal ventricular rate of 50 beats/ min and an atrial rate of 160 beats/min. Her ECG demonstrated complete AV block. There was no ischemic ST change. One minute after termination of exercise, stable $1: 1 \mathrm{AV}$ conduction reappeared with a rate of 50 beats/min (Fig. 1). Twenty-four-hour Holter monitoring disclosed paroxysmal 2:1 AV block at an atrial rate of 80 beats/min and a ventricular rate of 40 beats/min over the course of 30 minutes (17:30-18:00) (Fig. 2). Coronary angiography showed no stenosis of the right or left coronary arteries, and ergonovine provocation test was negative. 

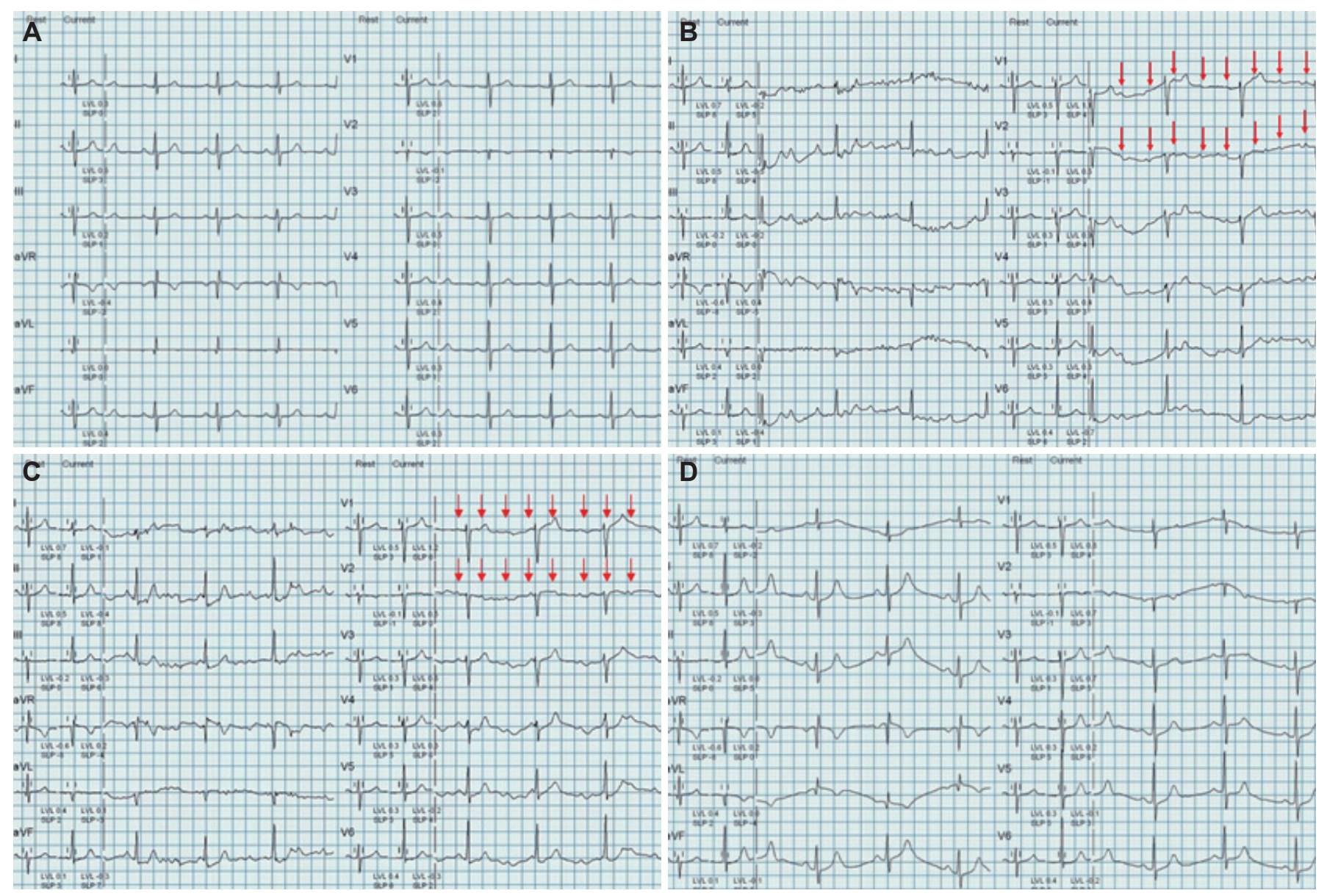

Fig. 1. Treadmill exercise test. A: at rest-normal AV conduction. B: stage 1-high degree AV block. C: stage 2-complete AV block. D: at recovery-normal AV conduction. AV: atrioventricular.

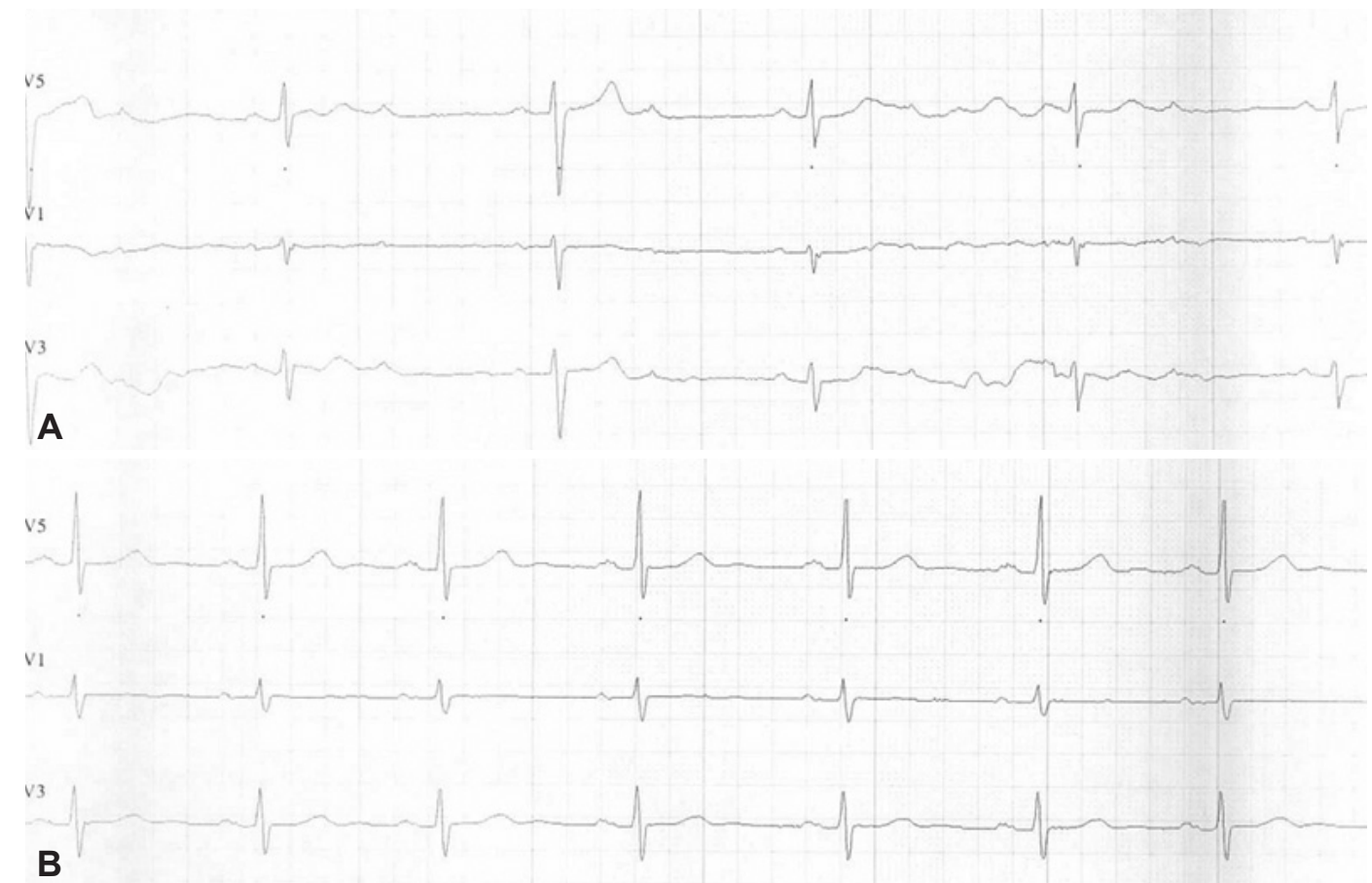

Fig. 2. Twenty-four-hour Holter monitoring. A: $2: 1$ AV block (atrial rate, 80 beats/min). B: normal AV conduction (atrial rate, 54 beats/min). AV: atrioventricular. 

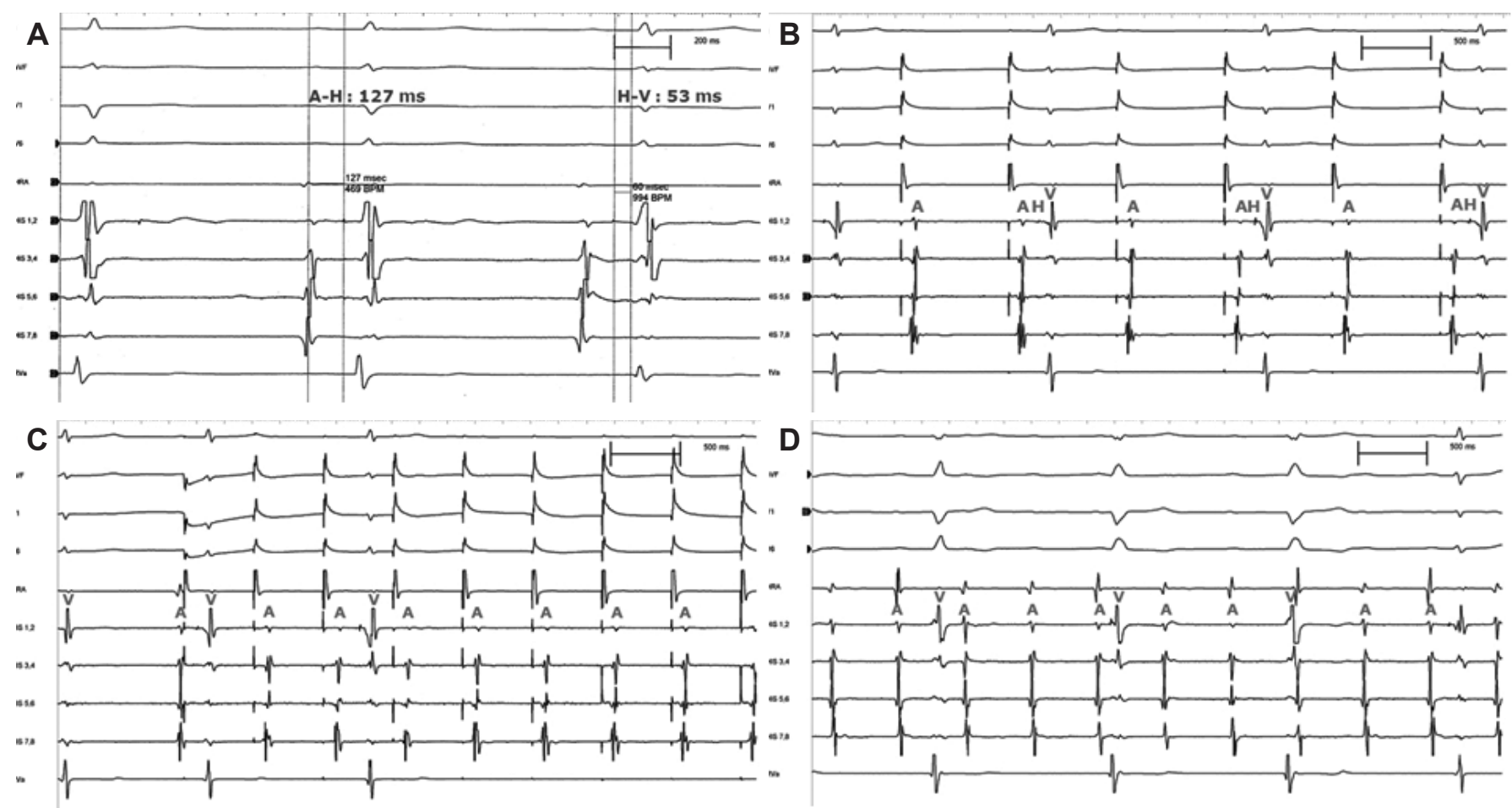

Fig. 3. Electrophysiologic study. A: basic sinus cycle length. B: HRA pacing at a cycle length of 780 msec-2 : 1 AV block. C: HRA pacing at a cycle length of 500 msec-high-grade 2 nd degree AV block. D: intravenous atropine induced sinus rate acceleration-complete AV block. HRA: high right atrium, AV: atrioventricular.

Electrophysiological studies were performed on the patient, while she was in a non-sedated state. Basic sinus cycle length was 1000 msec, right atrial-His bundle deflection interval was $127 \mathrm{msec}$, and His-bundle-ventricle deflection interval was 53 msec. On atrial pacing at cycle lengths of $780 \mathrm{msec}, 2: 1 \mathrm{AV}$ block occurred and, at a cycle length of $500 \mathrm{msec}$, progressed to high-grade 2nd degree AV block. The block site was proximal to the His bundle. Upon withdrawal of atrial pacing, $1: 1 \mathrm{AV}$ conduction was promptly restored. In addition, sinus rate acceleration induced by intravenous atropine $(0.04 \mathrm{mg} / \mathrm{kg}$ ) provoked complete AV block. Electrophysiological studies demonstrated rate-dependent intranodal AV block (Fig. 3). The patient received a permanent dual chamber (DDD) pacemaker and had no symptoms during the follow-up period.

\section{Discussion}

Exercise-induced AV block is rare in patients who exhibit normal $1: 1$ AV conduction at rest $(0.45 \%) .{ }^{12)}$ However, this kind of AV block has clinical implications, since it is related to the development of dyspnea and syncope. ${ }^{3)}$ Two mechanisms have been proposed for the development of exercise-induced AV block. First, in normal subjects, conduction velocity increases through vagolysis, and the refractory period decreases at the AV node. However, since the HisPurkinje system is not influenced by the autonomic nervous system, the refractory period is not significantly decreased. This difference in refractoriness between the AV node and the His-Purkinje system can induce conduction disturbances in subjects with an abnormal conduction system, but not in those with a normal conduction system. Most cases of exercise-induced AV block are induced through this mechanism and show tachycardia-dependent blocks due to the disease of the His-Purkinje system. ${ }^{45)}$ Second, exercise-induced AV block can be induced by the AV nodal ischemia. Such patients may complain of chest pain during treadmill exercise testing and may show ischemic changes on ECGs and atherosclerotic lesions on coronary angiograms.

It is difficult to explain our patient's AV block through the two forementioned mechanisms. Since blood pressures were normal during exercise testing, her exercise-induced AV block did not seem to result from an increase in parasympathetic activity. In addition, since stenotic or atherosclerotic lesions were not detected in coronary angiograms or in spasm studies, the correlation between myocardial ischemia and the AV block does not apply to this case. Kasaoka et al. ${ }^{6}$ ) reported a case of sympathetic pseudo-AV block due to a His-bundle parasystole masquerading as exercise-induced $2: 1$ AV block. Previous reports on exercise-induced AV block demonstrated that His bundle parasystole can be ruled out by revealing that AV block develops proximal to His bundle through electrophysiologic study, and that AV block develops again proximal to the His bundle 
when sinus tachycardia is induced by administration of atropine. ${ }^{3778)}$ The precise mechanisms for rate-dependent intranodal AV block remain to be explained.

We presented the case of a 67-year-old woman who developed exercise-induced AV block at the AV node level accompanied by dizziness and who showed symptomatic recovery after implantation of a pacemaker.

\section{References}

1. Ozder AB, Kůçükoğlu S, Doğar H, Karcier SM, Demiroğlu C. Exercise-induced AV block. Am Heart J 1989;117:1407-9.

2. Egred $M$, Jafary $F$, Rodrigues E. Exercise induced atrio-ventricular (AV) block: important but uncommon phenomenon. Int J Cardio/ 2004;97: 559-60.

3. Sumiyoshi M, Nakata Y, Yasuda M, et al. Clinical and electrophysiologic features of exercise-induced atrioventricular block. Am Heart J 1996; 132:1277-81.

4. Hemann BA, Jezior MR, Atwood JE. Exercise-induced atrioventricular block: a report of 2 cases and review of the literature. J Cardiopulm Rehabil 2006;26:314-8.

5. Choi JY, Kim HY, Rho TH, Kim JH. A case of paroxysmal atrioventricular block complicating Takayasu's Arteritis. Korean Circ J 1994;24:717-21.

6. Kasaoka Y, Ajiki K, Hayami N, Murakawa Y. His-bundle parasystole masquerading as exercise-induced 2:1 atrioventricular block. J Cardiovasc Electrophysiol 2001;12:965-7.

7. Pappas LK, Efremidis M, Sideris A, Letsas KP, Kounas SP, Kardaras F. Exercise-induced second-degree atrioventricular block. Int J Cardio/ 2006; $111: 461-3$.

8. Martí-Almor J, Cladellas M, Bruguera J. Atrioventricular block induced by exercise is not always infrahisian. Rev Esp Cardio/ 2005;58:1247-8. 